impotence, for example-is likely to be charged with emotional overtones. Such patients are best seen in a non-teaching clinic.

In the wards the difficulties are less. The interpatient relationships, from which emerges a sort of esprit de corps, seldom fail to release the patient from the inhibitions surrounding teaching. Curtains must be drawn and, as in outpatient clinics, candlewick dressing gowns or attractive turkish towelling used to diminish any embarrassment for the patient. Discussion on differential diagnosis, prognosis, and treatment is best carried out away from the patient's bed and out of earshot of other patients. But afterwards it is essential that the teacher returns to have a personal word with the patient, who may feel threatened by the mere fact that he has been excluded from the conversation.

The Department's suggested texts to be sent to prospective outpatients and inpatients of a teaching hospital are actually less inspiring than those already used by some teaching hospitals. No mention is made of the benefits that may accrue to the patient by attending a teaching hospital, something that is well appreciated in the United States and for which there is statistical evidence in this country. Even if it were not politic to mention this tactfully, patients know that students must be taught and doctors further trained. They appreciate too that most advances in clinical medicine emanate from hospitals where research and teaching are pursued. As the circular does indeed point out, they can be readily made to understand that they themselves are helping the advance of medicine.

1 Department of Health and Social Security, Teaching on Patients, $\mathrm{HM}(73) 8$.

\section{Sudden Whitening of Hair}

An event as dramatic as sudden whitening of hair is inevitably surrounded by a rich folk lore, to which exaggeration and inaccurate reporting have contributed. For over a century the experts have disputed whether it is ever possible, many stating categorically that hair once formed is dead and inaccessible to such a change. But in recent years several fully authenticated cases have been reported in which a mechanism is apparent.

J. E. Jelinek ${ }^{1}$ has recently reviewed many of the fascinating earlier reports from historical and literary sources, and A. J. Ephraim ${ }^{2}$ has given a full account of medical reports over the past 150 years. The historical cases that have come down to us nearly always occurred in dramatic circumstances. Even in Shakespeare's day sudden whitening of hair was attributed to shock. Sir Thomas More's hair apparently turned white during the night before his execution and General Gordon's during the final stages of the seige of Khartoum. Among other cases are some memorable for their symbolism, such as that of a man whose hair turned suddenly white when he woke up to find himself alone with an appropriately grizzly bear. Even more remarkable is the tale of a 24-year-old officer whose hair on only half of his beard and scalp turned white after a night of carnal dissipation with a mulatto. Even in 1940 a textbook is quoted as saying that rape of young girls is a common cause of sudden whitening. Many of these early reports are poorly authenticated, and though fairly rapid whitening of hair can be believed the exact timing and relationship to the reported cause has to be treated with considerable scepticism.

Various theories have been put forward to account for this phenomenon. Vitiligo and other depigmenting diseases may at times spread rapidly on the skin, may affect the hair, and may sometimes apparently be associated with emotional or neurological disorders. It can cause whitening of hair over some months, but could hardly cause overnight whitening of already pigmented hair. To account for rapid whitening it has been suggested that air bubbles may suddenly be forced into the hair matrix. Such a mechanism is responsible for the paler segments of the banded hair of pili annulati, but again it seems incredible that this could affect the whole hair shaft overnight, and it has not been convincingly demonstrated.

In alopecia areata regrowth of hair is often white, and no doubt this could account for some cases where the timing had not been carefully noted. However, the most plausible theory is that sudden whitening of hair is due to some disease process which selectively causes the dark hairs to be shed, sparing the white. Grey hair is an admixture of fully p'gmented and white hairs, and even as little as $5 \%$ or less of residual white hairs could make a respectable covering. That the white hairs are often spared in the patches of alopecia areata has long been known, ${ }^{3}$ and any dermatologist could expect to see this at least once or twice every year. Rarely alopecia areata may have an explosive onset involving the whole scalp, and in the past 15 years several cases have been recorded leading to sudden whitening of hair. ${ }^{14}$ It is noteworthy that an acute shock was not apparent in these cases. This is in accord with the current ideas that emotional factors are not primarily concerned in the pathogenesis of alopecia areata, though their precise role is still disputed, 56 for there is a lingering impression, unsubstantiated by accurate statistics, that acute emotional disturbances can provoke attacks of alopecia areata in a few patients. The difference in behaviour of white and dark hairs in alopecia areata must surely hold some clue to its aetiology.

1 Jelinek, J. E., Bulletin of the New York Academy of Medicine, 1972, 48, 1003.

2 Ephraim, A. J., Archives of Dermatology, 1959, 79, 228.

3 Sabouraud, R., Maladies du Cuir Chevelu, V. Les syndromes Alopéciques. Pelades t $t$ Alopécies en Aires. Paris, Mason, 1929.

Klingmüller, V. G., Dermatologica, 1958, 117, 84.

5 Macalpine, I., British fournal of Dermatology, 1958, 70, 117.

- Reinhold, M., British Medical fournal, 1960, 1, 846.

\section{Postoperative Empyema and Survival in Lung Cancer}

Despite advances in surgical technique and postoperative care the five-year survival rate in lung cancer remains disappointingly low. Retrospective examination of the notes of patients who have had the good fortune to live for five or more years after pneumonectomy can only rarely give clues to why surgery was successful. In most circumstances the great number of uncontrolled variables that are part of any random series of cancer cases are such as to render retrospective studies of little use. However, occasionally this type of study can be rewarding if an unequivocal event appears to have intervened between surgery and the expected progression of the tumour to widespread metastasis.

Empyema was a fairly common complication in the pioneering days of thoracic surgery, but during the last 20 